\title{
Reaction Synthesis of TiC and Fe-TiC Composites*
}

\author{
M. J. CAPALDI, A. SAIDI ${ }^{1)}$ and J. V. WOOD
}

Department of Materials Engineering and Materials Design, The University of Nottingham, University Park, Nottingham, NG7 2RD, UK. 1) Isfahan University of Technology, Isfahan, Iran.

(Received on June 19, 1996; accepted in final form on November 12, 1996)

\begin{abstract}
The combustion synthesis of $\mathrm{TiC}$ and $\mathrm{Fe}-\mathrm{TiC}$ by thermal explosion and self propagating high temperature synthesis has been investigated. The experimental methods are outlined and a method for the simultaneous measurement of wavefront velocity and maximum combustion temperature is described. The morphology of the products obtained is similar in both reaction modes. Values for the activation energy for each system were calculated and used to propose that the formation of $\mathrm{Fe}-\mathrm{TiC}$ occurs via the formation of a $\mathrm{FeTi}_{2}$ eutectic, liquid at the reaction temperature, which allows the transfer of carbon. In the TiC system the liquid phase is titanium.
\end{abstract}

KEY WORDS: combustion synthesis; SHS; titanium carbide.

\section{Introduction}

The terms combustion synthesis and self propagating high temperature synthesis refer to reactions which once initiated proceed in a self supporting manner without any external energy input. Two experimental procedures have been investigated in the present study; thermal explosion (TE) and normal self propagating high temperature synthesis (SHS), to examine the combustion synthesis in the titanium-carbon and the titanium-ironcarbon systems. The two reaction modes can be represented by the SHS diagram shown in Fig. 1. ${ }^{1)}$ TE involves rapid furnace heating until a reaction is initiated at several points simultaneously throughout an entire sample and SHS, the rapid heating of the surface of a sample to an initiate reaction at one point which then proceeds by the propagation of a combustion front through the length of the sample, this is represented schematically in Fig. 2.

General combustion synthesis reactions are characterised by high reaction temperatures up to $4000 \mathrm{~K}$, short reaction times, a few seconds for TE and combustion front propagation of up to $250 \mathrm{~mm} \mathrm{~s}^{-1}$ for SHS and in the case of SHS extremely large thermal gradients, $\sim 10^{5}-10^{6} \mathrm{~K} \mathrm{~m}^{-1}$. The majority of studies in the field of combustion synthesis have been concerned with reactions between transition metals and non-metals to form metal carbides, borides, silicides, hydrides and nitrides however many other materials such as intermetallics, oxides and superconducting phases can be produced. ${ }^{2-5)}$ The advantages of combustion synthesis over conventional powder metallurgy processes are the low energy requirements (sufficient to initiate reaction) compared to long high temperature sintering cycles, and the relative purity of the final products due to the expulsion of occluded gases and volatile impurities at high temperature. The economic advantages can however be offset by the need

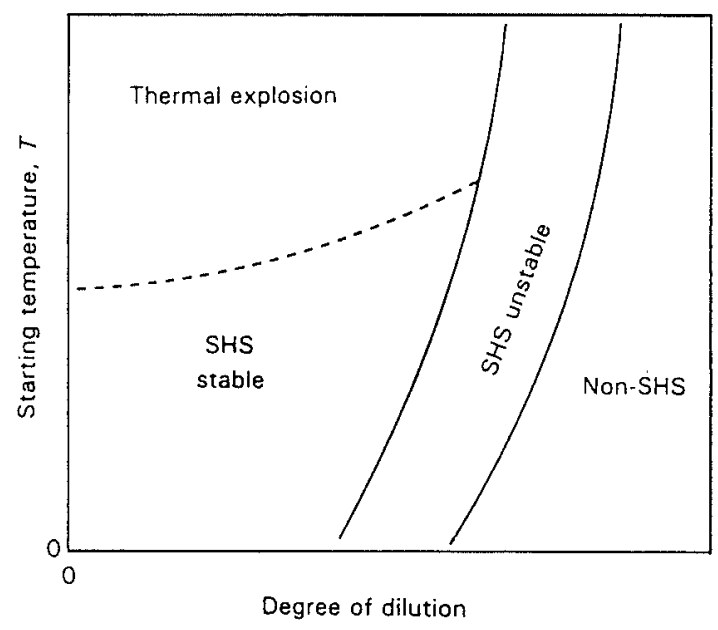

Fig. 1. SHS diagram.
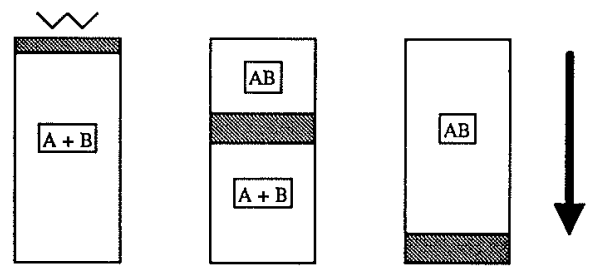

1

2

3

Fig. 2. Schematic representation of SHS process: arrow indicates direction of combustion from ignition (1), wave propagation (2) to completion (3).

\footnotetext{
* This paper was presented in the "International Workshop on Thermophysical Data for the Development of Mathematical Models of Solidification" held at Gifu City from 6-8 October, 1995.
} 
to start with elemental or partially alloyed reactants. Also the final product microstructure is difficult to control and is often porous through the volatilisation of impurities and density differences between reactants and products. The porosity can be reduced by reacting under pressure and fully dense components may be produced by the combination of combustion synthesis and hot isostatic pressing (HIP). ${ }^{6-8)}$ Materials made by combustion synthesis have found use in high wear applications, hard alloys and thermal spray powders where the inherent porosity of the powder makes grinding simpler.

\section{Theory}

The large negative heat of reaction usually associated with combustion synthesis gives the thermodynamic driving force for the process. This is given by the following relationship for the formation of $\mathrm{AB}$ from $\mathrm{A}$ and $\mathrm{B}$,

$$
\Delta H_{\text {reaction }}=\Delta H_{\mathrm{f}, \mathrm{AB}}-\left(\Delta H_{\mathrm{f}, \mathrm{A}}+\Delta H_{\mathrm{f}, \mathrm{B}}\right)
$$

which simplifies to the enthalpy of formation of the product phase, $\mathrm{AB}$, for reaction from elemental reagents. For the formation of titanium carbide from an elemental mixture of titanium and carbon as used for this study, the heat of reaction is the enthalpy of formation of $\mathrm{TiC}$, $\Delta H_{\mathrm{f}, 298}^{\circ}=-184 \mathrm{~kJ} \mathrm{~mol}^{-1}$. The heat of reaction for some systems particularly intermetallic compounds is not sufficient to sustain a combustion wave in an SHS process but reaction can be initiated with pre-heating of samples and such systems are usually studied by the thermal explosion route.

For the combustion process to be self propagating the evolution of exothermic heat must exceed that lost to the surroundings by radiative and convective processes. As combustion synthesis is fast and heating rates are high the reaction can be thought of as almost adiabatic. The maximum temperature attainable for any given system can be derived from the following expression,

$$
\Delta H_{\mathrm{r}, 298}^{\circ}=\int_{298}^{T_{\mathrm{ad}}} C_{\mathrm{p}} d T
$$

where $C_{\mathrm{p}}$ is the heat capacity of the products and $T_{\mathrm{ad}}$ is the maximum or adiabatic temperature. In practice however few systems can be thought of as truly adiabatic and the maximum recorded reaction temperature is usually several hundred degrees below the theoretical value. In the $\mathrm{TiC}$ reaction the adiabatic temperature is $3210 \mathrm{~K}$ but experimental temperatures measured in the present and other studies are of the order of $2500 \mathrm{~K}$.

\subsection{Wave Velocity Analysis}

For SHS reactions the velocity of the propagating wavefront can be used to determine a value for the activation energy, $E_{\mathrm{a}}$ for the process. A knowledge of this value gives an indication of the possible rate determining step for the reaction and therefore is useful for testing the validity of any proposed reaction mechanism. The method of wave velocity analysis outlined by Dunmead et al. ${ }^{9)}$ was used in this study. This method uses the relationship between the maximum reaction temperature and the measured wave front velocity according to the relationship,

$$
\frac{u^{2}}{T_{\mathrm{c}}^{2}}(1-x)=A e^{-E_{\mathrm{a}} / R T_{\mathrm{c}}}
$$

where $u$ is the wavefront velocity $\left(\mathrm{cm} \mathrm{s}^{-1}\right), T_{\mathrm{c}}$ is the maximum recorded temperature $(\mathrm{K}), R$ is the universal gas constant and $A$ is a complex constant term with contributions from the order of reaction, thermal diffusivity and heat capacity of the product and the heat of reaction. In order to determine a value for the activation energy it is necessary to be able to alter the two measured parameters. This can be achieved either by pre-heating of the sample or by the addition of an inert diluent, usually the product of the reaction which acts as a moderator. The second method was used for this study by adding varying quantities of prereacted $\mathrm{TiC}$ to the reaction mixtures. In the above relationship the term $(1-x)$ is used to take into account the effect on the heat of reaction caused by the addition of a weight fraction, $x$, of diluent. The activation energy is calculated from a plot of $\ln \left\{(1-x)^{1 / 2} u / T_{\mathrm{c}}\right\}$ vs. $1 / T_{\mathrm{c}}$ which has a gradient equal to $-E_{\mathrm{a}} / 2 R$.

\section{Experimental Procedure}

For both reaction modes the sample preparation was identical. This involved the milling together of titanium powder, carbon black and iron powder, for the $\mathrm{Fe}-\mathrm{Ti}-\mathrm{C}$ system and $\mathrm{TiC}$ for wave velocity measurements. Table 1 lists the specifications of the reagents used. The mixed powders were then compressed into pellets with a diameter of $10 \mathrm{~mm}$ and up to $25 \mathrm{~mm}$ in length. Compaction was uniaxial under pressures from 2 to $600 \mathrm{MPa}$.

\subsection{Thermal Explosion Mode}

Compacted samples contained in a graphite crucible were placed in an induction furnace. The furnace chamber was evacuated and backfilled with argon several times to ensure a low oxygen partial pressure. The samples were heated under an argon pressure of $0.7 \mathrm{~atm}$ until ignition was observed. The temperature attained during the explosion was monitored using a two colour pyrometer coupled to a data acquisition system capable of registering up to 500 measurements per second. A schematic diagram of the experimental apparatus used for this part of the study is shown in Fig. 3.

\subsection{Normal SHS Mode with Wave Velocity Measure- ment}

The apparatus used for this mode of reaction is shown in Fig. 4. It consisted of a steel glove box which could

Table 1. Specifications of reagents.

\begin{tabular}{lcl}
\hline & Particle size & \multicolumn{1}{c}{ Impurities (max. ppm) } \\
\hline $\mathrm{Ti}$ & $<45 \mu \mathrm{m}$ & $\mathrm{C}(100), \mathrm{Cl}(1700), \mathrm{Fe}(200)$ \\
& $<100 \mu \mathrm{m}$ & $\mathrm{N}(100), \mathrm{O}(1000)$ \\
$\mathrm{Fe}$ & $<500 \mu \mathrm{m}$ & \\
Carbon black & $<60 \mu \mathrm{m}$ & $\mathrm{Si}(1400), \mathrm{C}(200), \mathrm{P}(150), \mathrm{S}(150)$ \\
& $<1 \mu \mathrm{m}$ & \\
\hline
\end{tabular}




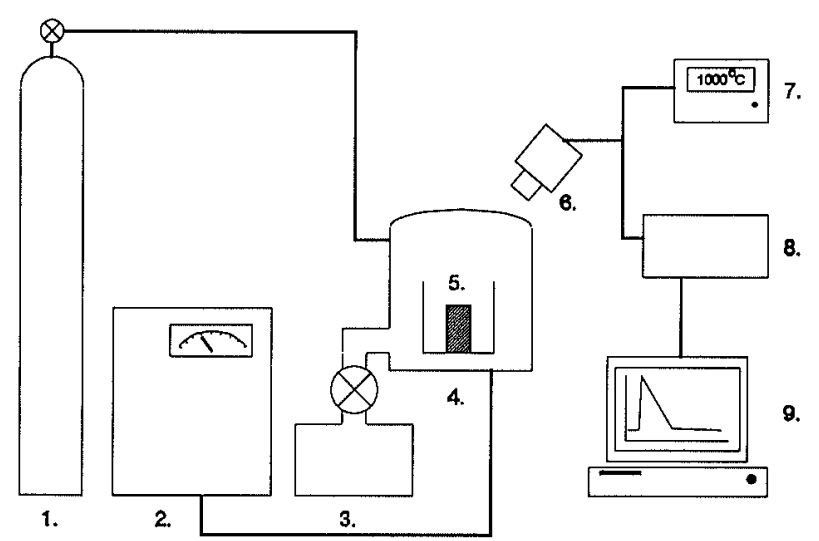

Fig. 3. Apparatus used for Thermal Explosion (TE) experiments. (1) argon supply, (2) power supply, (3) vacuum pump, (4) furnace chamber, (5) sample, (6) pyrometer, (7) temperature display, (8) data acquisition system, (9) PC.

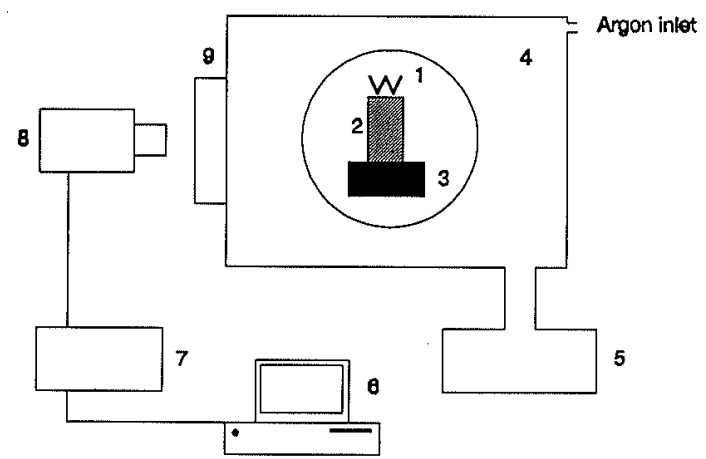

Fig. 4. Apparatus used for normal SHS experiments. (1) tungsten filament, (2) sample, (3) graphite stage, (4) glove box, (5) vacuum pump, (6) PC, (7) data acquisition system, (8) pyrometer, (9) quartz window.

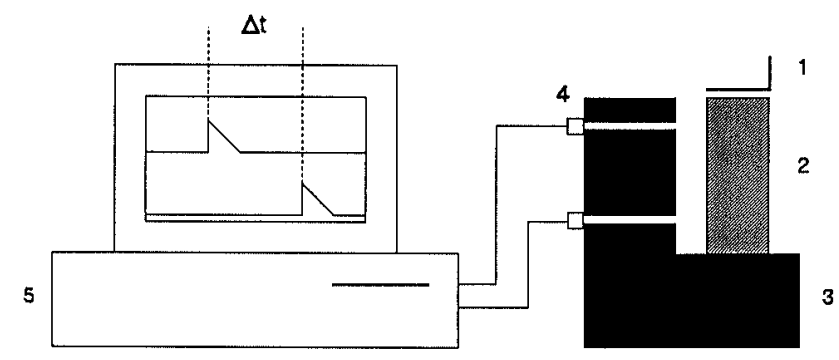

Fig. 5. Wave velocity measurement system: wave velocity, $u=x / \Delta t$. (1) tungsten filament, (2) sample, (3) graphite stage, (4) photodiode, (5) PC.

be evacuated and backfilled with argon to allow manipulation in an inert atmosphere. The compacted samples were placed on a graphite stage and positioned so that the top surface was $\sim 1-2 \mathrm{~mm}$ below a tungsten wire. The wire was resistively heated by the application of a direct current of $c a .20-30 \mathrm{~A}$. Once the reaction was seen to start the current was switched off and the sample left to react. The temperature of the combustion wave was recorded using the same data collection system as for the TE experiments with the pyrometer focused through a quartz window in the side wall of the glove box onto the midpoint of the sample. The rate of propagation of the combustion wave was measured by two photodiodes built into the graphite support stage. This is shown in Fig. 5.
The passage of the combustion front is detected by each diode and a signal sent to the data acquisition system. The time taken to pass between the two diodes can then be obtained and the speed calculated.

\section{Results and Discussion}

\subsection{Titanium-Carbon Reaction under Thermal Explo- sion Mode}

Different heating rates and titanium particle size were found to have a marked effect on the reactions observed in this system. Equiatomic mixtures of titanium and carbon with a titanium particle size in excess of $100 \mu \mathrm{m}$ could not be reacted in a self supporting manner even when heated to $1450^{\circ} \mathrm{C}$ at the maximum heating rate for the system of $350^{\circ} \mathrm{C} \mathrm{min}^{-1}$. This was due to the formation of a protective layer of $\mathrm{TiC}$ formed on the surface of the titanium particles by solid state diffusion. A self supporting thermal explosion was seen when the titanium particle size was reduced. With a heating rate of $350^{\circ} \mathrm{C}$ $\min ^{-1}$ the ignition of mixtures containing titanium with a particle size between 100 and $45 \mu \mathrm{m}$ took place at $1206^{\circ} \mathrm{C}$ and reached a maximum temperature of $2500^{\circ} \mathrm{C}$. When the titanium particle size was less than $45 \mu \mathrm{m}$ ignition occurred at $1166^{\circ} \mathrm{C}$ with a maximum temperature of $2610^{\circ} \mathrm{C}$. Mixtures containing the smallest titanium particles could not however be reacted when heating rate was reduced to $250^{\circ} \mathrm{C} \mathrm{min}^{-1}$. Only a partial reaction was observed at $1550^{\circ} \mathrm{C}$ due to the formation of $\mathrm{TiC}$ on the prior titanium particle surfaces by solid state diffusion. The effect of precompaction pressure was evidenced by the observation that uncompacted samples (loose powders) and samples compacted at $>10 \mathrm{MPa}$ could not be ignited and within this range, higher compaction pressures led to higher ignition temperatures.

The effect of these experimental parameters on the thermal explosion of titanium and carbon has been fully detailed in a previous publication. ${ }^{10)}$ For a self supporting process to occur the exothermic heat generated in one part or several parts of the sample must exceed that lost by conduction and radiation. When this situation occurs the temperature of the adjacent regions will increase sharply and promote a thermal explosion with rapid propagation of the reaction through the rest of the sample. This situation is more favourable when the rate of reaction in the region of interest is fast, allowing a rapid build up of exothermic heat before it can be dissipated. Any change in experimental parameters which cause an increase in the rate of reaction will therefore promote a thermal explosion. Hence the beneficial effects that are observed with small particle sizes and high heating rates. The precompaction pressure has an effect which is twofold, an increase in pressure allows more intimate contact between particles and thus increases reaction rate however this also increases the thermal conductivity of the sample and thus a point is reached where the rate of heat generation is matched by the dissipation by conduction and no local temperature rise is observed, hence there is no thermal explosion. Figure 6 shows the microstructure of $\mathrm{TiC}$ formed by thermal explosion. 


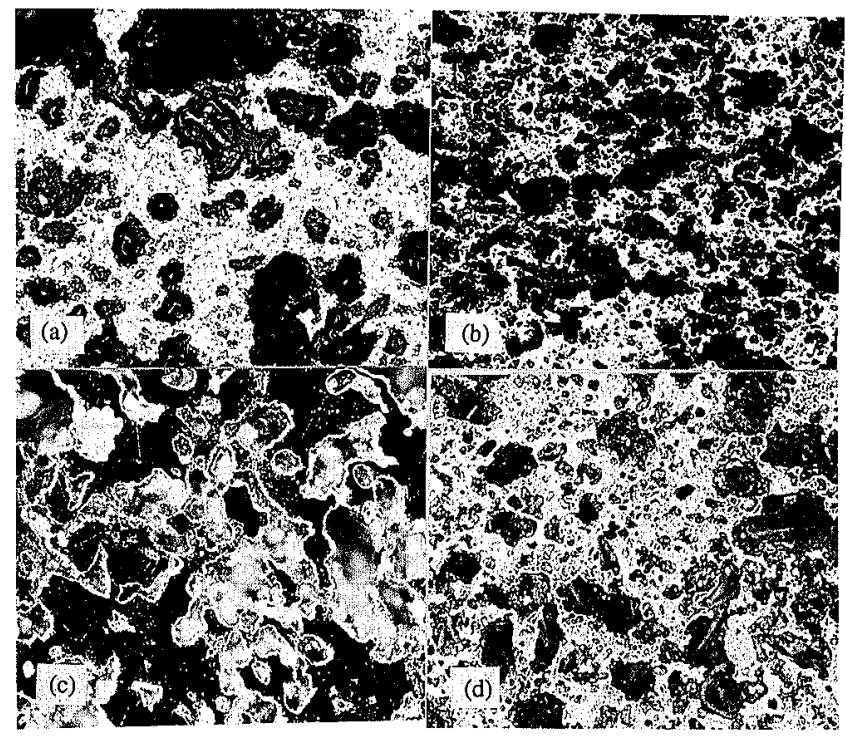

Fig. 6. Optical micrographs of structures of $\mathrm{TiC}$ formed by TE (a), SHS (b), Fe-TiC by TE (c) and SHS (d).

\subsection{Titanium-Carbon Reactions under Normal SHS Mode}

The ease of ignition and the formation of a stable combustion wave was found to be dependent on several experimental factors. The most successful reactions were those between titanium with a particle size of $<45 \mu \mathrm{m}$ and carbon black. Larger metal particles either made ignition impossible or resulted in unstable combustion and incomplete product formation. Inefficient mixing of the reagents also gave rise to an incomplete reaction. There was no evidence of the dual role played by the compaction pressure found in the thermal explosion mode. Well mixed loose powders could be ignited and there was no upper boundary at which combustion was not achieved. This was to be expected as the increase in thermal conductivity associated with higher compaction pressure is beneficial to the propagation of a combustion wave which relies on the advance of a pre-heating zone preceding the actual reaction zone. The structure of the product is similar to that formed by TE and is shown in Fig. 6.

Successful SHS reactions were also performed by using ratios of titanium and carbon which were not equiatomic. The cubic $\mathrm{TiC}_{x}$ phase is stable with the range $0.55<x<$ 1.00 and can be formed as a pure phase within these stoichiometries by SHS. Table 2 lists the variation in lattice parameter for SHS formed $\mathrm{TiC}_{x}$. Figure 7 shows $\mathrm{XRD}$ traces of phases made from mixtures corresponding to $\mathrm{TiC}_{0.55}, \mathrm{TiC}_{0.75}$ and $\mathrm{TiC}_{0.95}$. Complete conversion to the carbide is seen in all cases with no evidence of free titanium. The variation in lattice parameter is in agreement with literature ${ }^{11)}$ Reactions of mixtures containing a greater excess of titanium or a twofold excess of carbon were unsuccessful.

A value for the activation energy for the reaction between an equiatomic mixture of titanium and carbon was obtained using the method of Dunmead et al. outline above. The addition of $\mathrm{TiC}$ as a diluent in quantities from 0 to $25 \mathrm{wt} \%$ allowed the temperature of combustion
Table 2. Calculated cell dimensions ${ }^{12,13)}$ for $\mathrm{TiC}_{x}$.

\begin{tabular}{cc}
\hline Stoichiometry & Cubic cell dimension $(\AA)$ \\
\hline $\mathrm{TiC}_{0.55}$ & $4.283(2)$ \\
$\mathrm{TiC}_{0.75}$ & $4.312(2)$ \\
$\mathrm{TiC}_{0.95}$ & $4.317(2)$ \\
\hline
\end{tabular}

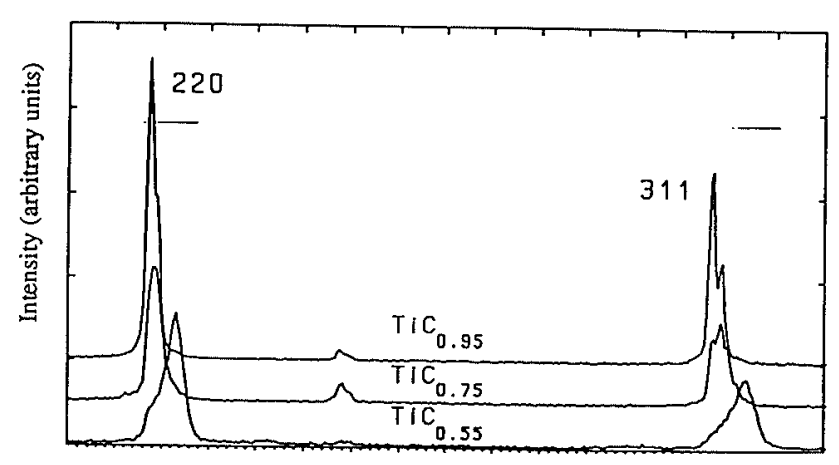

$2 \theta \rho \rightarrow$

Fig. 7. Region of $\mathrm{X}$-ray diffraction traces for $\mathrm{TiC}_{0.55}, \mathrm{TiC}_{0.75}$ and $\mathrm{TiC}_{0.95}$ showing variation of lattice parameter.

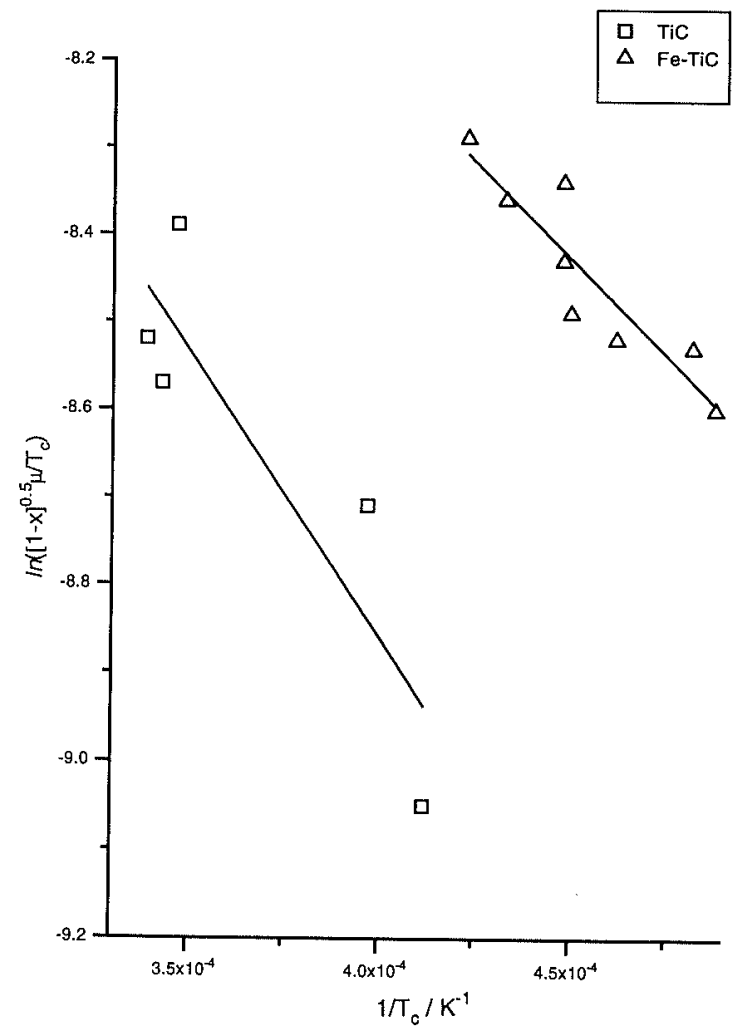

Fig. 8. Plots of $\ln \left\{(1-x)^{1 / 2} \mu / T_{\mathrm{c}}\right\}$ vs. $1 / T_{\mathrm{c}}$ used for the calculation of activation energy of $\mathrm{TiC}$ and $\mathrm{Fe}-\mathrm{TiC}$ by SHS.

and the rate of propagation of the combustion front to be varied. These parameters ranged from $2678^{\circ} \mathrm{C}$ and $5.9 \mathrm{~mm} \mathrm{~s}^{-1}$ for an undiluted sample to $2155^{\circ} \mathrm{C}$ and $3.3 \mathrm{~mm} \mathrm{~s}^{-1}$ for a sample containing $25 \mathrm{wt} \% \mathrm{TiC}$. The calculated activation energy of $109 \mathrm{~kJ} \mathrm{~mol}^{-1}$ agrees well with the quoted value for the dissolution of carbon in molten titanium of $117 \mathrm{~kJ} \mathrm{~mol}^{-1}$ allowing for experimental error, since the measurement of the peak combustion temperature by pyrometry is subject to a error of a least $\pm 20^{\circ} \mathrm{C}$. This data is shown in Fig. 8 . 


\subsection{Titanium-Carbon-Iron Reactions under the Ther- mal Explosion Mode}

The effect of the addition of iron to a titanium-carbon mixture on the ignition and combustion temperatures during TE has been discussed in a previous paper. ${ }^{10)}$ Small iron additions lowered the ignition temperature while leaving the combustion temperature unchanged and reactions using large titanium particles, $250-500 \mu \mathrm{m}$ and low heating rates, $200^{\circ} \mathrm{C} \mathrm{min}{ }^{-1}$ were possible. This was attributed to the formation, by solid state diffusion of the eutectic compound $\mathrm{FeTi}_{2}$ which has a melting point of $1085^{\circ} \mathrm{C}$. It is the melting of this eutectic which allows the dissolution of carbon and the subsequent reaction and precipitation of $\mathrm{TiC}$. The heat evolved by the formation of $\mathrm{TiC}$ allows the reaction to self propagate. This mechanism is supported by the formation of liquid droplets in a sample heated to just below ignition before being quenched. As the maximum combustion temperature is below the melting point of either of the metals involved the eutectic is the only possible liquid phase.

Increasing the iron content had little effect on temperatures with only a slight increase in ignition temperature found at $80 \mathrm{wt} \% \mathrm{Fe}$. The combustion temperature remained invariant with iron addition until $60 \mathrm{wt} \%$ $\mathrm{Fe}$ where it was found to drop from $c a .2500$ to $1442^{\circ} \mathrm{C}$ at $80 \mathrm{wt} \% \mathrm{Fe}$. Mixtures with more than $80 \mathrm{wt} \% \mathrm{Fe}$ did not react in a self supporting manner.

\subsection{Titanium-Carbon-Iron Reactions under the Normal SHS Mode}

The eutectic compound $\mathrm{FeTi}_{2}$, the melting of which is thought to be the origin of the liquid phase responsible for the transport of carbon in the reactions described above occurs at $71 \mathrm{wt} \% \mathrm{Ti}$. The activation energy associated with the reaction between $71 \mathrm{wt} \% \mathrm{Ti}, 29 \mathrm{wt} \%$ $\mathrm{Fe}$ and carbon in equiatomic ratio with titanium was determined as above. TiC was again used as an inert diluent and gave rise to maximum combustion temperatures from $2090-1777^{\circ} \mathrm{C}$ and combustion front velocities from $6.0-4.1 \mathrm{~mm} \mathrm{~s}^{-1}$ for $\mathrm{TiC}$ additions from $0-16 \mathrm{wt} \%$. The value obtained of $76 \mathrm{~kJ} \mathrm{~mol}^{-1}$ (see Fig. 8) was considerably lower than that for the reaction between titanium and carbon. This is consistent with a less energetic rate determining step for the process and therefore supports the mechanism involving the molten eutectic.

XRD analysis of the products shows peaks which can be attributed to TiC and elemental iron (Fig. 9). The participation of iron in the reaction seems to be to form the low melting eutectic required for carbon transfer and does not take part in any further chemical reaction assuming there is no excess carbon present. The product is a matrix of iron containing particles of titanium carbide. The morphology of the product is similar irrespective of the mode of reaction and is shown in Fig. 6.

Increasing the iron content was found to both lower the maximum combustion temperature and the wave velocity. This is shown in Fig. 10. Complete combustion was observed for samples containing $60 \mathrm{wt} \% \mathrm{Fe}$, partial combustion for $70 \mathrm{wt} \% \mathrm{Fe}$ and no combustion at

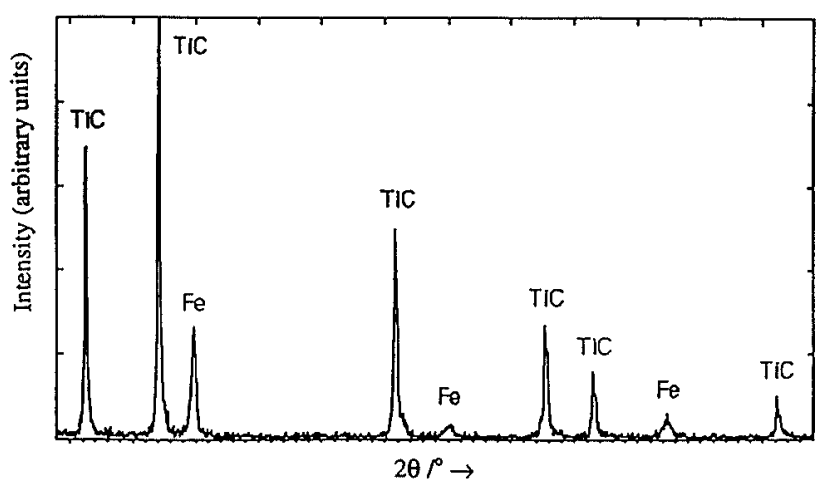

Fig. 9. X-ray diffraction trace of Fe-TiC produced by SHS.

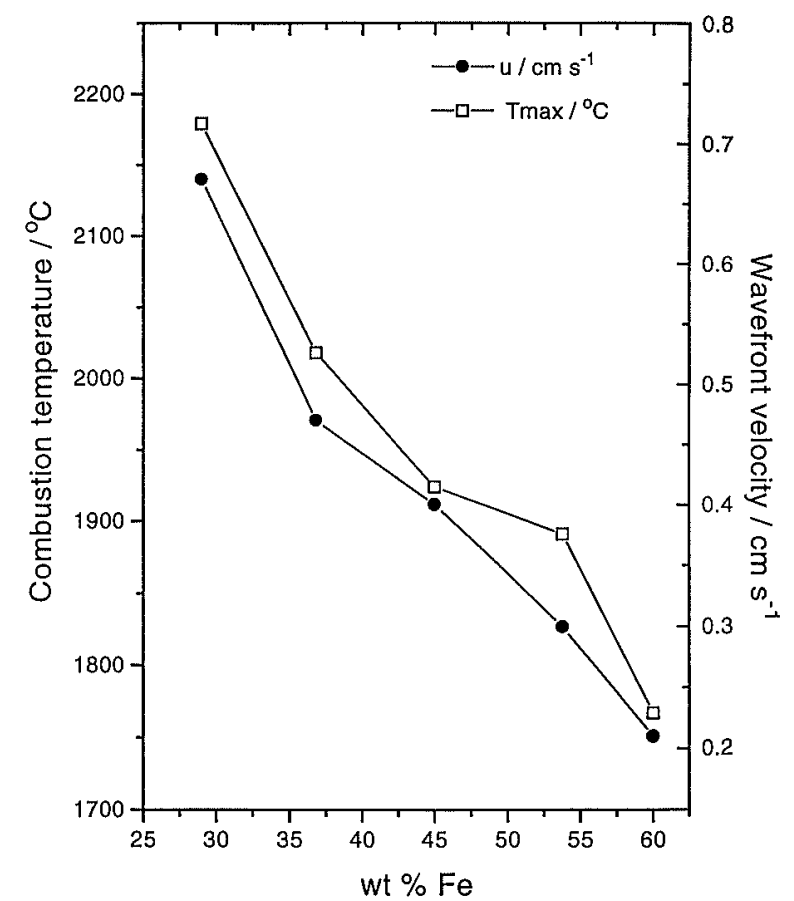

Fig. 10. Variation of maximum temperature and wave velocity with increasing $\mathrm{Fe}$ content.

$>70 \mathrm{wt} \% \mathrm{Fe}$.

\section{Conclusions}

Combustion synthesis of $\mathrm{TiC}$ and $\mathrm{Fe}-\mathrm{TiC}$ produces similar products irrespective of the mode of reaction. These are characterised by a porous microstructure which is caused by the expulsion of volatile impurities and density differences in the reactant mixture. Full conversion to product is observed and substoichiometric titanium carbide can be produced from a suitable mixture. The addition of iron leads to a product which has TiC contained in a matrix of iron. A limit of between 70 and $80 \mathrm{wt} \% \mathrm{Fe}$ exists beyond which a self supporting reaction cannot readily be achieved.

The mechanism of reaction between titanium and carbon is thought to be dominated by the diffusion of carbon into molten titanium resulting in the formation of $\mathrm{TiC}$ in solution which then precipitates on cooling. The addition of iron allows the formation of a low melting eutectic which corresponds to $\mathrm{FeTi}_{2}$. This has a melting point of $1085^{\circ} \mathrm{C}$ and is thought to be the liquid phase responsible for the diffusion of carbon. The formation 
of $\mathrm{TiC}$ takes place by precipitation from this eutectic, leaving a product of $\mathrm{TiC}$ in iron. The activation energies calculated for both systems support this mechanism.

\section{Acknowledgments}

This work was supported by an EPSRC ROPA award and undertaken in collaboration with $\mathrm{J}$. Kellie and $\mathrm{J}$. Ellis of the London and Scandinavian Metallurgical Company, Rotherham, UK.

\section{REFERENCES}

1) N. Sato and Z. A. Munir: Proc. of Symp. on High Temperature Materials, Vol. 9-18, ed. by W. B. Johnson and R. A. Rapp, Pennington, NJ, (1990), 99.

2) H. C. Yi and J. J. Moore: J. Mater. Sci., 25 (1990), 1159.

3) J. W. McCauley: Ceram. Eng. Sci. Proc., 11 (1990), 1137.
4) J. Subrahmanyam and M. Vijayakumar: J. Mater. Sci., 27 (1992), 6249.

5) A. G. Merzhanov: Adv. Mater, 2 (1990), 570

6) Y. Miyamoto and K. Tanihata: New Horizons for Mater, 4 (1995), 387

7) Y. Miyamoto and M. Ohyanagi: New Functionality Mater., C (1993).

8) P. D. Zavitsanos and J. Morris: Ceram. Eng. Sci. Proc., 4 (1983), 624.

9) S. D. Dunmead and Z. A. Munir: J. Am. Ceram. Soc., 1 (1992), 180.

10) A. Saidi, A. Chrysanthou and J. V. Wood: J. Mater. Sci., 29 (1994), 4993.

11) E. K. Stroms: The Refractory Carbides, ed. by J. L. Margrav, Academic Press, NY, (1967), 8.

12) D. Louer and M. Louer: J. Appl. Cryst., 5 (1972), 271.

13) A. Boultif and D. Louer: J. Appl. Cryst., 24 (1991), 987. 